TEMAS EMERGENTES

\title{
La protección de los derechos sexuales y reproductivos en tiempos de covid-19
}

\author{
The protection of sexual and reproductive rights in times of Covid-19
}

\author{
Alejandra Isabel Pérez Ceballos iD \\ Universidad Autónoma de Chile
}

\begin{abstract}
RESUMEN En este artículo se analizarán las principales afectaciones y amenazas que, a consecuencia de la pandemia provocada por la enfermedad covid-19, evidencian los derechos sexuales y reproductivos a nivel mundial, al ser afectado su ejercicio como resultado de las medidas tomadas por los Estados en resguardo de la población. Producto del estudio, se establecen los mecanismos y directrices capaces de reforzar aún más su debida protección, basados en las recomendaciones de los principales organismos internacionales. En paralelo, se describirán las medidas y protocolos que en Chile se han establecido en la protección de los derechos sexuales y reproductivos en período de pandemia por covid-19. Es mediante un esfuerzo mancomunado, entre los actores de la sociedad civil y política, en conjunto con las instancias internacionales, que se debe atender con preocupación el actual escenario en que los derechos fundamentales se encuentran inmersos, para lograr su recuperación en el pleno ejercicio de los derechos.
\end{abstract}

PALABRAS CLAVE Derechos sexuales y reproductivos, derechos humanos, derecho internacional, covid-19, estado de excepción constitucional.

ABSTRACT This article will analyze the main effects and threats that, because of the pandemic caused by the Covid-19 disease, evidence sexual and reproductive rights worldwide, affecting their exercise as a result of the measures taken by the States to protect the population and product of this study, to establish those mechanisms and guidelines that are capable of further reinforcing their due protection, based on the recommendations that the main International Organizations have made. The measures and protocols that Chile has established in the protection of sexual and reproductive rights in a period of pandemic by Covid-19 will be described in parallel. It is through a joint effort, among the actors of civil and political society, in conjunction with international bodies, that the current scenario in which fundamental rights are immersed must be addressed with concern and their recovery in the full exercise of them. 
KEYWORDS Sexual and reproductive rights, human rights, international law, Covid-19, state of constitutional exception.

\section{Introducción}

En el contexto de la actual pandemia y los estrictos confinamientos que durante el año 2020 ha debido enfrentar la humanidad, a consecuencia del virus SARS-CoV2, causante de la enfermedad covid-19, se presenta un complejo escenario en el ejercicio de los derechos fundamentales, producto de las diversas restricciones que las autoridades de cada país y los organismos a nivel internacional válidamente han debido implementar en resguardo de la población. Es entonces materia de este artículo el estudio de aquellas afectaciones y, como consecuencia, la debida protección que desde su especificidad evidencian los derechos sexuales y reproductivos.

Inmersos en una emergencia sanitaria mundial, los derechos sexuales y reproductivos se ven comprometidos con mayor notoriedad, debido a las limitaciones que en distintos ámbitos involucran a la libertad personal, efectuadas con la clara finalidad de resguardar la vida, la salud y la seguridad individual de todas y cada una de las personas que habitan nuestro planeta.

El objetivo de este artículo es demostrar la manera en que se han visto afectados a nivel mundial los derechos sexuales y reproductivos, como resultado de expansión de la pandemia por covid-19. También se propone, a partir de las recomendaciones internacionales, un reforzamiento en su debida protección y promoción, desde la mirada específica de estos derechos humanos universalmente reconocidos.

$\mathrm{Al}$ respecto, el Instituto Interamericano de Derechos Humanos recalca la idea de que «es fácil así comprobar que los derechos reproductivos son parte integral e indivisible de los derechos humanos, pero como la ampliación y profundización de los derechos humanos en general y de los derechos humanos de las mujeres en particular, es un proceso dinámico» (IIDH, 2008: 24).

En primer lugar, se desarrollará en los capítulos iniciales la conceptualización y reconocimiento a nivel internacional de los derechos sexuales y reproductivos, para luego contextualizar el marco normativo nacional. En los siguientes acápites se analizarán los principales pronunciamientos que han publicado los organismos internacionales respecto del covid-19, relacionados directamente con los derechos sexuales y reproductivos. Luego, a partir de ellos, se establecen las recomendaciones aplicables por los Estados, con el objeto de fortalecer su promoción y garantía, orientando en este último punto el análisis principalmente hacia cuatro grupos de mayor riesgo: las mujeres y el impacto diferenciado que ha presentado la pandemia en sus vidas, con especial preocupación en la violencia de género; las personas que se identifican con la comunidad LQTBIQ+ y el refuerzo al momento de instaurar medidas que apoyen 
y orienten un trato adecuado por parte de instituciones públicas y organizaciones sociales; hombres y mujeres en el acceso oportuno y adecuado a la salud reproductiva, y, por último, las personas portadoras de $\mathrm{VIH} /$ sida.

\section{Conceptualización y regulación normativa relativa a los derechos sexuales y reproductivos}

\section{Desarrollo conceptual de los derechos sexuales y reproductivos}

Los derechos sexuales y reproductivos constituyen el reconocimiento de un conjunto de libertades relacionadas a la sexualidad humana, los cuales, a su vez, emanan del reconocimiento universal a la igualdad y no discriminación entre hombres y mujeres, la autonomía, la integridad y la dignidad inherente a toda persona.

La vivencia de la sexualidad humana es sin duda un proceso complejo, no ajeno en algunas ocasiones a reparos de tipo ético. Este tema no sólo involucra elementos biológicos o aprendizaje de valores, sino que también la correcta aplicación de la normativa legal.

La sexualidad debe ser entendida como un proceso dinámico, que tiende a cambiar conforme al desarrollo y evolución del ser humano, por ello resulta ser cambiante e identitaria. La sexualidad es una condición del ser humano que lo integra por completo y que le permite manifestarse como su fuero interno lo diga y oriente. Para Saeteros, Sanabria y Pérez (2014: 299), «la sexualidad humana, contendría componentes biológicos y sicológicos bien determinados; en consecuencia, es el resultado de la construcción social».

Dentro de las libertades en el pleno ejercicio de los derechos sexuales y reproductivos, encontramos la autonomía que poseen las personas para adoptar decisiones sobre su sexualidad, como el decidir si se quiere o no iniciar la vida sexual; tener una vida sexual activa; expresar libre y autónomamente la orientación sexual; poder vivir la sexualidad sin violencia, coacción, abuso, explotación o acoso; formar familia; tener hijos; poder acceder a información y educación en los temas planteados, y un adecuado acceso a la salud sexual y reproductiva.

El primer germen de reconocimiento internacional de los derechos sexuales y reproductivos en un sentido genérico y poco profundizado se estableció en la Conferencia Internacional de Derechos Humanos, celebrada en Teherán el 13 de mayo de 1968, que estableció en su Declaración 16: «La comunidad internacional debe seguir velando por la familia y el niño. Los padres tienen el derecho humano fundamental de determinar libremente el número de sus hijos y los intervalos entre los nacimientos».

Décadas después, en la Conferencia Internacional de Población y Desarrollo (CIPD), celebrada en el Cairo en 1994, se discutieron con mayor profundidad y firmeza los temas relacionados a los derechos humanos de la población, la salud sexual 
y reproductiva, la igualdad entre géneros y el derecho a planificar el tipo de familia, el número de hijos que se desea tener y el espaciamiento entre ellos.

Según Corrêa y Betânia Ávila (2003: 21), el término derechos sexuales fue introducido como estrategia de negociación en la CIPD en 1994, para que los derechos reproductivos fueran garantizados en el texto final de la Declaración y Programa de Acción de El Cairo; la inclusión del término sexual radicalizaba el lenguaje, de forma que al conceder su supresión, se negociaba la permanencia de «derechos reproductivos». Por eso el término derechos sexuales no aparece en el documento final del Programa de Acción de El Cairo.

En consecuencia, los derechos sexuales y reproductivos se consagraron internacionalmente, como se mencionó, en la Conferencia Internacional de Población y Desarrollo, que fue reafirmada al año siguiente en la IV Conferencia Mundial sobre la Mujer de 1995, en Beijing.

Según el párrafo 7.3 del citado Programa de Acción de El Cairo:

Los derechos reproductivos abarcan ciertos derechos humanos que ya están reconocidos en leyes nacionales, documentos internacionales sobre derechos humanos y en otros documentos aprobados por consenso. Estos derechos se basan en el reconocimiento del derecho básico de todas las parejas e individuos a decidir libre y responsablemente el número de hijos, el espaciamiento de los nacimientos y a disponer de la información y de los medios para ello, así como el derecho a alcanzar el nivel más elevado de salud sexual y reproductiva. También incluye el derecho a adoptar decisiones relativas a la reproducción sin sufrir discriminación, coacciones o violencia, de conformidad con lo establecido en los documentos de derechos humanos.

El reconocimiento internacional de los derechos sexuales y reproductivos se profundizó en la IV Conferencia Mundial sobre la Mujer del año 1995. De conformidad con lo previsto en el párrafo 96 de la Declaración y Plataforma de Acción de Beijing:

Los derechos humanos de las mujeres incluyen su derecho a tener control sobre las cuestiones relativas a su sexualidad, incluida su salud sexual y reproductiva, y a decidir libremente respecto de esas cuestiones sin verse sujeta a coerción, discriminación o violencia.

La autora Rosalind Petchesky (1999) señala que el desarrollo - aunque incipiente- del concepto de derechos sexuales, inicialmente sólo fue posible de forma negativa, o sea, enunciando el derecho de no ser objeto de abuso o explotación, en el sentido paliativo del combate a las violaciones.

Para la autora, es necesario que el desarrollo de los derechos sexuales sea ampliado hacia un concepto positivo, que vaya más allá del combate a las discriminaciones y abusos cometidos contra las minorías sexuales, incluidas ahí las mujeres que no se encuadran en las formas dominantes de su género. Así refuerza el planteamiento de 
que los derechos sexuales deben englobar las llamadas titularidades (entitlements) afirmativas, concluyendo que las titularidades afirmativas y negativas son los dos caras de la misma moneda: «No puedo gozar de mi cuerpo sexual si estoy constantemente sometida al temor, digamos, de un abuso» (Petchesky, 1999: 16).

Cuando se estableció internacionalmente el reconocimiento de los derechos sexuales y reproductivos, la Organización de Naciones Unidas reforzó las ideas planteadas en las Conferencias presididas, ampliando su reconocimiento, al hacer la siguiente recomendación:

La salud reproductiva entraña la capacidad de disfrutar de una vida sexual satisfactoria y sin riesgos y de procrear, y la libertad para decidir hacerlo o no hacerlo, cuándo y con qué frecuencia. Esta última condición lleva implícito el derecho del hombre y la mujer a obtener información y de planificación de la familia de su elección, así como otros métodos para la regulación de la fecundidad que no estén legalmente prohibidos, y acceso a métodos seguros, eficaces, asequibles y aceptables, el derecho a recibir servicios seguros y adecuados de atención de la salud que permitan los embarazos y los partos sin riesgos y den a las parejas las máximas posibilidades de tener hijos sanos. [...] la definición de salud reproductiva [...] incluye también la salud sexual, cuyo objetivo es el desarrollo de la vida y de las relaciones personales y no meramente el asesoramiento y la atención en materia de reproducción y de enfermedades de transmisión sexual. ${ }^{1}$

A partir del contexto internacional descrito, es necesario robustecer la premisa esencial de que los derechos sexuales y reproductivos son propios de la naturaleza humana y que en su extensión no son privativos de las mujeres, al ser todas las personas titulares de estos derechos. Desde la especificidad, podemos mencionar a los niños, niñas y adolescentes que, según su madurez y desarrollo progresivo, adquieren un discernimiento para tomar decisiones sobre su vida y su cuerpo; los adultos y principalmente los adultos mayores, por lo que es necesario derribar mitos respecto de su sexualidad; personas con capacidades diferentes; miembros de pueblos originarios y etnias, por lo que debe reconocerse la especial manera de percibir y vivir la salud sexual y reproductiva, de acuerdo con su propia cosmovisión y la comunidad LGTBIQ+.

En el reconocimiento de los derechos sexuales y reproductivos, se deben mencionar aquellos tratados internacionales que amparan estos derechos desde su especialidad: la Recomendación General Comité CEDAW 12 y 19 en contra de la violencia hacia la mujer; la Recomendación 15, para evitar la discriminación contra la mujer en las estrategias nacionales de acción preventiva y lucha contra el sida del año 1990;

1. «Informe de la Conferencia Internacional sobre la Población y el Desarrollo», Naciones Unidas, A/ CONF.171/13/Rev.1, 1995, p. 37, disponible en https://bit.ly/3aq6jJc. 
la Observación General 24 sobre la eliminación de todas las formas de discriminación contra la mujer; la Observación General 28 sobre la igualdad entre hombres y mujeres del Comité de Derechos Humanos de las Naciones Unidas del año 2000; la Observación General 14 sobre el Derecho a la Salud del Pacto Internacional de los Derechos Económicos, Sociales y Culturales del año 2000; la Observación General 5, del Comité de Derechos del Niño, sobre medidas generales de aplicación de la Convención sobre los Derechos de los Niños de 2003.

Para la Organización Mundial de la Salud (OMS), en conjunto con la Organización Panamericana de la Salud (OPS), los derechos sexuales y reproductivos

forman parte del derecho humano a la salud integral, comprendida como un estado general de bienestar físico, mental y social, y no de mera ausencia de enfermedades o dolencias. El derecho a la salud reproductiva trata de todos los aspectos relacionados con el sistema reproductivo, así como la capacidad de disfrutar de una vida sexual satisfactoria y sin riesgos, y de tener la libertad para decidir si procrear o no, cuándo hacerlo y con qué frecuencia. ${ }^{2}$

Habiendo contextualizado el concepto de los derechos sexuales y reproductivos y su reconocimiento en el plano internacional, en una aproximación a lo que se analizará en el acápite siguiente, se planteará el contexto normativo vigente en Chile en materia de protección de estos derechos. Adelantamos que, a pesar de no existir un reconocimiento expreso constitucional o legal que contemple de manera específica y exclusiva los derechos sexuales y reproductivos, nuestro ordenamiento jurídico protege y reconoce aquellas temáticas que los componen, por lo que la protección de los derechos sexuales y reproductivos se enmarca y refuerza en el respeto universal de los derechos esenciales que emanan de la naturaleza humana y que se consagran en nuestro plexo normativo, de conformidad con nuestra Carta Fundamental y los tratados internacionales ratificados por el país que se encuentran actualmente vigentes.

\section{Contexto normativo de los derechos sexuales y reproductivos en Chile}

En Chile no existe en la actualidad un reconocimiento normativo intencionado hacia los derechos sexuales y reproductivos dentro de la especialidad que merecen. A diferencia de otros países de la región, como Colombia, México, Ecuador o Paraguay, Chile no ha constitucionalizado su protección de manera específica, a pesar de forjar el intento en diversos proyectos presentados al Legislativo, por incorporarlos de manera especial o más explícita en nuestra norma nacional y sobre todo constitucional.

2. «CIDH exhorta a todos los Estados a adoptar medidas integrales e inmediatas para respetar y garantizar los derechos sexuales y reproductivos de las mujeres», Organización de los Estados Americanos, 23 de octubre de 2017, disponible en https://bit.ly/2Wp8goe. 
El 19 de octubre del año 200o, en el Gobierno del presidente Ricardo Lagos, se intentó buscar la positivización en esta materia, presentando ante la Cámara de Diputados, el proyecto de ley marco sobre derechos sexuales y reproductivos (Boletín 2.608-11), pero fue finalmente archivado el año 2016, encontrándose en su etapa inicial de revisión por la Comisión de Salud de la Cámara de Diputados.

El 7 de octubre de 2004 ingresó a la Cámara de Diputados el proyecto de ley que «Reforma la Constitución Política de la República con el objeto de establecer una nueva garantía constitucional en materia de derechos sexuales reproductivos», archivado el año 2009 (Boletín 3.207-07). También se intentó el año 2006 tramitar el proyecto de ley que «Establece la garantía constitucional del derecho a la libertad sexual y reproductiva», que fue archivado en 2016.

En ambos proyectos se proponía una reforma constitucional al artículo 19 de la Constitución, que consagra los Derechos y Deberes Constitucionales, agregando un nuevo numeral segundo, que establecería un primer avance al reconocer y proteger el derecho a la libertad sexual y reproductiva, consagrando en particular el derecho a la mujer comprendiendo la libertad y autodeterminación para elegir los métodos científicamente más apropiados para la prevención y planificación del embarazo.

Se debe destacar el proyecto de reforma constitucional, iniciado por mensaje a tramitación el 6 de marzo de 2018, de la entonces presidenta Michelle Bachelet, para modificar la Constitución Política de la República (Boletín 11.617-07) que incorporaba, dentro del nuevo texto propuesto, el reconocimiento expreso de derechos provenientes de la intimidad y desarrollo sexual y reproductivo de las personas, así como el derecho a la personalidad en sentido amplio. Lamentablemente, dicho proyecto de reforma, que venía siendo antecedido por un largo proceso de trabajos en comisiones y cabildos ciudadanos, presentado los últimos días del mandato presidencial, ad portas de comenzar el actual mandato del presidente Sebastián Piñera, no logró concretar su vida normativa.

De dicho proyecto se rescatan los articulados propuestos en materia de reconocimiento de los derechos sexuales y reproductivos, originados desde un plano de la igualdad y dignidad de la persona humana, como atributos intrínsecos de todos y todas.

Tal como se indicó en el comienzo de este capítulo, el hecho de no encontrarse reconocidos expresamente en nuestra Carta Fundamental o en una ley especial, no impide que los derechos sexuales y reproductivos sean promovidos por nuestro ordenamiento jurídico en concordancia con el reconocimiento y cautela de los derechos esenciales que emanan de la naturaleza humana y cuyo ejercicio involucra los aspectos más íntimos y personales derivados de las decisiones que las personas toman respecto de su vida, su cuerpo y decisiones. Motivo por el cual, en período de pandemia por covid-19, es a partir de ese reconocimiento de los derechos fundamentales que se pueden reforzar medidas para abordar a los derechos sexuales y reproductivos. 
Nuestra actual Carta Fundamental asegura de manera amplia a todas las personas derechos fundamentales universalmente reconocidos, resguardados en complemento por el plexo internacional, de los cuales puede subsumirse la protección de toda persona en sus decisiones sexuales y reproductivas, como parte de las libertades y autonomía.

Los derechos humanos universalmente reconocidos que se encuentran incorporados hoy en nuestra Constitución son aquellos derechos inherentes a la dignidad humana, que se traduce en libertades e igualdades, como la dignidad de la persona humana (artículo 1); el derecho al reconocimiento de la familia como núcleo fundamental de la sociedad y el deber del Estado de promover y protegerla (artículo 1); el derecho a la vida y a la integridad física y síquica de la persona, la protección mandatada al legislador de la vida del que está por nacer y la prohibición de todo apremio ilegítimo (artículo 19, numeral primero); la igualdad ante la ley e igual protección de la ley en el ejercicio de los derechos, así como la prohibición de toda forma de discriminación arbitraria, reforzando que hombres y mujeres son iguales ante la ley (artículo 19, numerales segundo y tercero); el derecho a participar con igualdad de oportunidades en la vida nacional (artículo 1); el derecho a la protección y respeto de la vida privada y la honra de la persona y su familia, sumada a la protección de los datos personales (artículo 19, numeral cuarto); el derecho a la libertad de conciencia y libertad de culto (artículo 19, numeral sexto); el derecho a la protección de la salud y garantizar su acceso (artículo 19, numeral noveno); el derecho a la educación (artículo 19, numeral décimo); el derecho a la libertad de emitir opinión (artículo 19, numeral duodécimo); el derecho a la libertad de trabajo y su protección y la prohibición de cualquier discriminación que no se base en la capacidad, o i idoneidad personal. (artículo 19, numeral decimosexto); y, por último, la protección de la esencialidad de los derechos, que se traducen en la no afectación en su esencia de los derechos reconocidos constitucionalmente en concordancia con el reconocimiento internacional vigente en nuestro país (artículo 19, numeral 26. ${ }^{\circ}$ ).

En el plano internacional, Chile ha suscrito acuerdos generales y específicos en temáticas de derechos sexuales y reproductivos, que fundan las bases para adoptar ciertas decisiones gubernamentales o políticas de Estado en la materia. ${ }^{3}$ Como pilar esencial sin duda se encuentran la Declaración Universal de Derechos Humanos (1948); el Pacto Internacional de Derechos Civiles y Políticos (1966) y sus protocolos facultativos (1966 y 1989), y el Pacto Internacional de Derechos Económicos, Sociales y Políticos (1966) y su protocolo facultativo (2008). En la región se fortalecen dichos preceptos bajo el alero de la Convención Americana de Derechos Humanos (1969) y la Convención Interamericana para Prevenir, Sancionar y Erradicar la Violencia Contra la Mujer, Belém do Pará (1994).

3. Listado confeccionado a partir de la información actualizada del sitio web de la Biblioteca del Congreso Nacional. 


\section{La protección de los derechos sexuales y reproductivos en tiempos de covid-19}

Dentro de las libertades en el pleno ejercicio de los derechos sexuales y reproductivos, se establecen, según las descripciones que los tratados internacionales hacen en sus cuerpos normativos la autonomía que poseen las personas para adoptar medidas libre y responsablemente sobre su sexualidad y vida reproductiva, como consecuencia del ejercicio de las libertades que como seres humanos poseemos en el reconocimiento de nuestra dignidad e igualdad, como, por ejemplo: el decidir si se quiere iniciar la vida sexual; definir la identidad sexual e identidad de género; decidir los compañeros o compañeras sexuales que tendrá; si se quiere tener una vida sexual activa; el acceso a métodos de anticoncepción o mecanismos de planificación familiar; acceso a información y tratamiento por enfermedades de transmisión sexual; el ejercicio de la vida privada e intimidad; establecer formas de comunicación y protección de sus datos personales; expresar libre y autónomamente la orientación sexual; poder vivir la sexualidad sin violencia, coacción, abuso, explotación o acoso; decidir formar una familia, optar por un vínculo afectivo; tener hijos; interrumpir un embarazo; o acceder a mecanismos de reproducción asistida. Todas estas libertades se ven hoy limitadas bajo el contexto de encierro dentro de una cuarentena y el estado de excepción constitucional decretado.

A continuación, se analizarán los cuatro escenarios que muestran más evidencia haber sido afectados durante la pandemia, por lo que requieren una protección reforzada por parte de los Estados y los organismos internacionales ante la vulneración o amenaza en su ejercicio legítimo y pleno para sus titulares. Se organizan en cuatro grupos, atendiendo la vulneración a que pueden ser objeto, en un momento tan complejo para la humanidad como la actual emergencia sanitaria: la afectación que sufren las mujeres y el impacto diferenciado que ha presentado la pandemia en sus vidas, con especial preocupación en la violencia de género; las personas que se identifican con la comunidad LGTBIQ+ y el refuerzo al momento de establecer medidas que apoyen y orienten un trato adecuado por parte de instituciones públicas y organizaciones sociales; los hombres y las mujeres que buscan acceder de manera oportuna y adecuada a la salud reproductiva, en especial a mecanismos de control de natalidad; por último, las personas portadoras de VIH/sida en la entrega efectiva de tratamientos médicos, evitando un desmedro en su estado de salud.

\section{Violencia y discriminación en contra de mujeres y niñas}

En este punto se analizará la manera en que la actual pandemia del covid-19 produce consecuencias negativas en la salud de mujeres y niñas, junto con propiciar un escenario hostil que ha provocado un aumento en la violencia contra la mujer y la 
afectación de su salud. Esto es motivo de preocupación extrema de las autoridades, sumado a la preocupación de los Estados por la calidad de vida de los habitantes a consecuencia de los efectos de la crisis sanitaria, en la salud pública, sus repercusiones socioeconómicas y la afectación en la economía y pérdida de puestos de trabajos.

El Comité de Naciones Unidas para la Eliminación de la Discriminación contra la Mujer (Comité CEDAW) ha hecho un llamado firme a los Estados miembros de la Convención sobre la Eliminación de Toda las Formas de Discriminación contra la Mujer (Convención CEDAW) a actuar de manera conjunta con la comunidad internacional en la protección de los principios de igualdad de género, no discriminación y la feminización de la pobreza, consecuencias todas de la actual crisis pandémica.

Lo anterior requiere la colaboración de todos los actores de la sociedad civil, en apoyo y preservación de los derechos de niñas y mujeres que se ven afectados producto del covid-19. Para estos efectos, el Comité estableció una guía de apoyo y procedimientos, ${ }^{4}$ invocando a los Estados parte de la Convención CEDAW sus obligaciones de proteger a las mujeres y actuar de manera rápida y eficaz ante la amenaza de su integridad física, síquica y su salud, reforzando la premisa de que

si bien muchos Estados consideran restricciones a la libertad de movimiento y al distanciamiento físico necesarias para prevenir el contagio, tales medidas pueden limitar desproporcionadamente el acceso de las mujeres a atención médica, refugios seguros, educación, empleo y vida económica. Los efectos se agravan para grupos desfavorecidos de mujeres y mujeres en situaciones de conflicto $u$ otras situaciones humanitarias.

La guía de orientaciones y lineamientos rectores establece directrices necesarias de robustecer para los Estados, en colaboración con la sociedad civil y la comunidad internacional, destacando para efectos de este acápite:

- Abordar el impacto de la pandemia en la salud de la mujer. Disminuir los sesgos de género en la asignación de recursos y la desviación de fondos durante las pandemias, que agrava las ya existentes desigualdades de género.

- Proteger a mujeres y niñas de la violencia de género, pues durante el encierro se encuentran en mayor riesgo de sufrir violencia doméstica, sexual, económica, sicológica y otras formas de violencia por parte de parejas abusivas, familiares y cuidadores, y en comunidades rurales.

- Reforzar el apoyo socioeconómico a las mujeres, producto de la crisis del covid-19, que afecta negativamente a las mujeres como consecuencia de encontrarse en empleos mal remunerados y en ocasiones informales, temporales $\mathrm{u}$

4. «Guidance Note on CEDAW and Covid-19», Comité de Naciones Unidas para la Eliminación de la Discriminación contra la Mujer, 2020, disponible en https://bit.ly/3h27iRo. 
otras formas de empleo precarias, especialmente en la ausencia de protección social.

- Fortalecer la respuesta institucional, la difusión de información y la recopilación de datos. Los Estados deben fortalecer y coordinar los mecanismos nacionales para responder con eficacia a las consecuencias del covid-19, propendiendo a la difusión correcta y actualizada de información científicamente precisa y transparente, sobre todo en aquellas materias que se traduzcan en riesgos de género del covid-19 y las medidas para los servicios de salud y apoyo disponibles para las mujeres y las niñas.

Para la Organización Panamericana de Salud (OPS), el covid-19 agrava los riesgos de la violencia contra la mujer como consecuencia del ambiente hostil que provocan las medidas de confinamientos y distanciamientos en los hogares, que aumentan las probabilidades de violencia hacia las mujeres, de sus hijos e hijas. A lo anterior se suma el escaso contacto que tienen con sus familiares y amigos, ya que en el contexto de cuarentenas evitan recurrir a ellos en búsqueda de apoyo y protección ante la violencia. Destaca la Organización que «los sistemas de salud desempeñan una función importante en garantizar que los servicios para las mujeres que han sufrido violencia sigan siendo accesibles durante el brote de covid-19».5

En complemento a las consecuencias negativas que presenta el covid-19 en mujeres y niñas, se evidencia el acceso limitado a los servicios básicos de salud sexual y reproductiva, incluyendo aquellos destinados a mujeres que sufren violencia, motivo por el cual la OPS refuerza la necesidad de potenciar los esfuerzos por parte de los sistemas de salud, destacando que desempeñan una función importante en garantizar que los servicios para las mujeres que han sufrido violencia sigan siendo accesibles durante la pandemia del covid-19, proponiendo una serie de medidas aplicable por los Estados para combatir la violencia contra la mujer durante la emergencia sanitaria.

A su vez, hace un llamado a los Gobiernos y las autoridades a cargo de las políticas públicas para incluir en sus planes de preparación y respuesta a la pandemia servicios esenciales para combatir la violencia contra la mujer, logrando su efectividad y accesibilidad.

También invita a reforzar la entrega de información que efectúan los establecimientos de salud, que incluya centros de atención de crisis por violencia, líneas telefónicas de ayuda y albergues, por lo que es necesaria la disponibilidad de los servicios a distancia.

También hace un llamado a que los prestadores de servicio de salud ofrezcan asistencia de manera empática, mejorando su seguridad y logrando conectarlas con los

5. «Covid-19 y violencia contra la mujer: Lo que el sector y el sistema de salud pueden hacer», Organización Panamericana de la Salud, 7 de abril de 2020, disponible en https://bit.ly/3rbYLQc. 
servicios de apoyo. Se plantean las posibilidades de telemedicina para abordar de manera segura la violencia contra la mujer.

Por último, propone mejorar los canales de contacto con familiares y amigos cercanos en búsqueda de apoyo, que sean reforzados por los servicios locales y los planes de seguridad, pudiendo establecer personas claves (amigos, vecinos, parientes o un albergue) ante quien pueda acudir en caso de tener que abandonar el hogar para preservar su seguridad.

Para la Comisión Interamericana de Mujeres (OEA-CIM), el covid-19 ha provocado un impacto diferenciado en la vida de las mujeres, recalcando la urgencia en el actuar de los Estados para incorporar y contemplar financiamiento para atender las necesidades de las mujeres en los planes y mecanismos de mitigación de la pandemia. Indican que

la emergencia derivada del covid-19 está provocando impactos específicos sobre las mujeres y profundizando las desigualdades de género existentes, tanto al interior de los hogares como fuera de ellos, en los hospitales y centros sanitarios, en el trabajo y también en la política. Esta realidad requiere que la participación igualitaria de las mujeres en las decisiones y el enfoque de género sean elementos centrales de las políticas de mitigación y recuperación de la crisis. ${ }^{6}$

Mostrando preocupación por el aumento de la violencia en contra de mujeres y niñas, la Comisión establece el siguiente diagnóstico, producto del confinamiento que obliga a las mujeres y niñas a estar encerradas en el hogar. Advierte que el confinamiento obliga a las mujeres a estar encerradas con la persona que las maltrata, generado por los conflictos domésticos que aumentan en tiempo de convivencia en pandemia, motivo por el cual es fundamental reforzar las líneas de apoyo y atención contra la violencia, centros de orientación y atención sicológica y jurídica. Advierte que las denuncias se dificultan en la práctica a causa de las cuarentenas y restricciones sanitarias y de libre circulación. Por otro lado, las niñas se ven mayormente violentadas, sobre todo sexualmente, ya que como consecuencia del confinamiento se ven más expuestas a abusos y violencia, por lo que los Estados deben promover su seguridad y apoyo para minimizar los riesgos, llamando la atención la sobreexposición tecnológica, que incrementa la violencia en internet (ciberviolencia).

Como medida de refuerzo, la Comisión propone que la tecnología se transforme en un medio facilitador para la interposición de denuncias «a través de medios como la telefonía inteligente y mensajería silenciosa, comisarías virtuales, botones de pánico, geolocalización, e incluso el uso de las redes sociales». ${ }^{7}$

6. «Covid-19 en la vida de las mujeres: Razones para reconocer los impactos diferenciados», Comisión Interamericana de Mujeres, 2020, p. 3, disponible en https://bit.ly/38lLtrP.

7. Comisión Interamericana de Mujeres, «Covid-19 en la vida...», 11. 
En relación con lo descrito, resulta preocupante la información que el Gobierno de nuestro país transparentó en sus últimos anuncios, evidenciando el aumento de las cifras en temas de violencia intrafamiliar en período de pandemia, en comparación con 2019. La violencia entre familiares y contra el género femenino aumenta en tiempos de cuarentena, según las cifras obtenidas de las llamadas telefónicas hechas por las víctimas.

El Ministerio de la Mujer y Equidad de Género informó que durante los primeros meses de pandemia (marzo y abril) «las denuncias por violencia intrafamiliar han disminuido entre $12 \%$ y $20 \%$ desde el inicio de la pandemia. Sin embargo, los llamados al Fono 1455 de Orientación han aumentado en $80 \%$ en el mismo periodo». ${ }^{8}$ Esto se debe a que no todas las denuncias o llamados reportando situaciones de violencia intrafamiliar se judicializan o se concretan en una denuncia ante la autoridad persecutora respectiva.

En voz de del Ministerio Público y Carabineros de Chile, sólo en el mes de marzo de 2020, en comparación al mismo mes del año pasado, las denuncias realizadas ante Carabineros por violencia intrafamiliar disminuyeron en $14 \%$, mientras que los llamados al 149 aumentaron en $20 \%$; mientras que el número de denuncias ante la Fiscalía presentó una disminución del $20 \%{ }^{9}$

Por otra parte, el delito de femicidio evidenció un aumento significativo «de $250 \%$ en los ingresos, lo que según el ente rector se explica por el aumento de este ilícito en su carácter frustrado, subiendo de 4 delitos en marzo de 2019 a 18 en marzo de 2020»..$^{10}$

Es evidente que el encierro de las víctimas y la convivencia sin escape son factores que evitan formalizar las denuncias, al no contar con la movilidad plena para hacerlo o no poder recurrir a algún recinto en busca de ayuda o resguardo, o lisa y llanamente no contar con un círculo de apoyo inmediato. Se han generado por este motivo otros mecanismos de ayuda, como ciertas «frases clave» para activar protocolos de asistencia, como por ejemplo la frase «mascarilla 19», que utilizan las mujeres que están siendo víctimas de violencia al momento de comprar un producto en una farmacia.

El actual Gobierno del presidente Sebastián Piñera ha implementado un programa de reforzamiento, a través de medidas de prevención y protección, en la continuidad de atención en los casos de violencia intrafamiliar y de género, además de

8. «Ministra de la Mujer oficia a Fiscal Nacional por denuncias de violencia intrafamiliar durante pandemia», Ministerio de la Mujer y Equidad de Género, 15 de mayo de 2020, disponible en https://bit. ly/2WpPMwF.

9. «Violencia intrafamiliar en tiempos de cuarentena: Comisión de la Mujer analiza cifras», Senado.cl, 9 de abril de 2020, disponible en https://bit.ly/2WzAXY9.

10. Shirley Berndt, «Fiscalía informa que durante marzo aumentaron los femicidios frustrados y bajaron las denuncias por violencia intrafamiliar», Emol.com, 3 de abril de 2020, disponible en https://bit. ly/38gUFO2. 
coordinación y orientación para ir en ayuda de las actuales víctimas y quien se sienta amenazada por hechos de violencia. El actual «Plan de contingencia de atención, protección y reparación ante los efectos del coronavirus» tiene como objetivo central:

velar por la continuidad de la atención, protección y reparación de mujeres víctimas y posibles víctimas de violencia en la red del Servicio Nacional de la Mujer y Equidad de Género en sus distintas instancias, así como proteger a los funcionarios que operan dichos servicios e implementar una fuerte difusión y comunicación de material de uso público a través de diversos canales de comunicación gubernamentales u otros que permitan cubrir la mayor población posible para enfrentar esta crisis sanitaria, especialmente para las mujeres de nuestro país que puedan o estén en riesgo de sufrir violencia. ${ }^{11}$

\section{Personas pertenecientes a la comunidad LGTBIQ+}

Es necesario recalcar la afectación que pueden a que pueden verse expuestas las personas del colectivo LGTBIQ+. Destaca la preocupación de la Comisión Interamericana de Mujeres, que indicó que pueden verse mayormente afectadas al momento de acceder a ciertos servicios y medios de apoyo, debido a la discriminación a que podrían ser víctimas, derivadas de los estereotipos de género.

Naciones Unidas, en colaboración con expertos y expertas internacionales en materia de derechos humanos, refuerzan el llamado a los Estados y otros actores de la sociedad civil y política a tener presente, de manera urgente, las repercusiones del covid-19 en las personas lesbianas, gay, bisexuales, transgénero o de género diverso al diseñar, implementar y evaluar las medidas preventivas y reactivas hacia la pandemia.

Se destaca la premisa planteada por Naciones Unidas en su Declaración de Expertos en Derechos Humanos en el Día Internacional contra la Homofobia, Transfobia y Bifobia (2020), al evidenciar que

las deficiencias en el respeto y garantía de las obligaciones derivadas del derecho a la vida de las personas LGBT son prácticamente generalizadas en muchas partes del mundo; estas deficiencias afectan también a los esfuerzos de recopilación de datos, la asignación de recursos y el apoyo que se brinda a la sociedad civil. Como consecuencia de lo anterior, la lucha contra la pandemia no se lleva a cabo en un plano de igualdad. ${ }^{12}$

11. «Plan de contingencia de atención, protección y reparación ante los efectos del coronavirus», Servicio Nacional de la Mujer y la Equidad de Género, disponible en https://bit.ly/3nzHi1E.

12. «Covid-19: El sufrimiento y la resiliencia de las personas LGBT deben ser visibles e informar de las acciones de los Estados», Oficina del Alto Comisionado de Naciones Unidas para los Derechos Humanos, 14 de mayo de 2020, disponible en https://bit.ly/3h2eLjo. 
Entre las recomendaciones que hace la OEA a los Estados en respuesta a la pandemia, en consideración a las personas adultos mayores, hace un llamado a

atender especialmente a las personas que están en situación de desigualdad estructural y discriminación por pertenecer a grupos LGTBI, atendiendo especialmente a las poblaciones mencionadas en el artículo. 5 de la Convención Interamericana sobre la Protección de Derechos de las Personas Mayores. ${ }^{13}$

En Chile, la Ley 20.609, conocida como «Ley Zamudio», tiene por objetivo fundamental instaurar un procedimiento judicial que permita restablecer de manera eficaz el imperio del derecho cuando se cometa un acto de discriminación arbitraria, obligando a cada uno de los órganos de la Administración del Estado a que, dentro del ámbito de su competencia, elabore e implemente políticas destinadas a garantizar a toda persona, sin discriminación arbitraria, el goce y ejercicio legítimo de sus derechos y libertades.

El artículo 2 de la ley entrega una definición de discriminación arbitraria, que entiende como toda distinción, exclusión o restricción que carezca de justificación razonable, indicando los criterios distintivos, efectuada por agentes del Estado o particulares, y que cause privación, perturbación o amenaza en el ejercicio legítimo de los derechos fundamentales establecidos en la Constitución Política de la República o en los tratados internacionales sobre derechos humanos ratificados por Chile y que se encuentren vigentes, incluyendo entre sus criterios discriminatorios el sexo, la orientación sexual, la identidad y expresión de género.

La orientación sexual, la identidad de género y la expresión de género son aspectos del derecho a la vida privada de las personas que el derecho protege en observancia a las obligaciones internacionales. La Comisión Interamericana de Derechos Humanos ha sostenido que

la orientación sexual constituye un componente fundamental de la vida privada de un individuo que debe estar libre de interferencias arbitrarias y abusivas por el ejercicio del poder público, en la ausencia de razones de mucho peso [...] el derecho a la privacidad protege el derecho a determinar la propia identidad y a formar relaciones personales y familiares en base a esa identidad, aunque la misma no sea aceptada o tolerada por la mayoría. Así, la orientación sexual, la identidad de género y la expresión de género son componentes fundamentales de la vida privada de las personas [...] el derecho a la vida privada garantiza esferas de la intimidad que el Estado ni nadie puede invadir, tales como la capacidad para desarrollar la propia personalidad y aspiraciones y determinar su propia identidad, así como campos de actividad de

13. "Guía práctica de respuestas inclusivas y con enfoque de derechos ante el covid-19 en las Américas», Organización de los Estados Americanos, 2020, p. 26, disponible en https://bit.ly/2KlGkaW. 
las personas que son propios y autónomos de cada quien, tales como sus decisiones, sus relaciones interpersonales y familiares y su hogar. ${ }^{14}$

En temas de salud sexual, el Colegio Médico de Chile es crítico en indicar que un inadecuado sistema de salud puede provocar que las personas lesbianas, gay, bisexuales, trans e intersexo no concurran a los centros hospitalarios por el temor a protagonizar tratos discriminatorios y ser atendidos por personas que desconocen las necesidades específicas de la población:

La crisis sanitaria llega en un momento donde aún no se cuenta con un protocolo de atención a personas trans que emane desde el Ministerio de Salud, sumado a que los centros de la red pública que poseen policlínicos de salud trans, se han conformado por voluntades locales y trabajo de la sociedad civil. Lo anterior genera incertidumbre en la continuidad de estos programas, considerando tanto ingresos como controles a las personas que demanden estas necesidades específicas de la comunidad. En Chile existe una deuda por parte del Estado frente a la falta de protocolos que eliminen las barreras de atención en salud a la diversidad sexual y de género. ${ }^{15}$

\section{Acceso a la salud reproductiva}

A causa de la dictación de cuarentenas obligatorias por parte de la autoridad, los derechos reproductivos se ven visiblemente irrumpidos ante nuevas circunstancias. La pandemia tendría implicancias directas en las decisiones que tanto personas como parejas adoptarían respecto del desarrollo de su vida sexual y reproductiva y, como consecuencia, la determinación de tener hijos o no, dadas las condiciones de salud y posibles contagios.

Respecto de la ejecutoriedad de medidas reproductivas, a nivel mundial se ha visto alterado en la cotidianeidad el derecho que poseen las personas para decidir de manera libre y responsable sobre el hecho de convertirse en madre o padre. La decisión, que hoy se ve influenciada en la práctica no sólo por la decisión de la persona o parejas, sino por externalidades como el Covid-19 o las medidas dispuestas por la autoridad, que condicionan el escenario en el cual se desarrolla este derecho reproductivo, así como en el resguardo y oportunidad de poder acceder a una atención a la salud de calidad durante el embarazo, el parto y el posparto.

Para el Consejo Económico y Social de Naciones Unidas:

14. «Orientación sexual, identidad de género y expresión de género: algunos términos y estándares relevantes», Consejo Permanente de la Organización de los Estados Americanos, CP/CAJP/INF. 166/12, 23 de abril de 2012, disponible en https://bit.ly/34q7ncc.

15. "Género y salud en tiempos de covid», Colegio Médico de Chile, mayo de 2020, disponible en https://bit.ly/3p8Vj6O. 
el derecho a la salud está estrechamente vinculado con el ejercicio de otros derechos humanos y depende de esos derechos, que se enuncian en la Carta Internacional de Derechos, en particular el derecho a la alimentación, a la vivienda, al trabajo, a la educación, a la dignidad humana, a la vida, a la no discriminación, a la igualdad, a no ser sometido a torturas, a la vida privada, al acceso a la información y a la libertad de asociación, reunión y circulación. Esos y otros derechos y libertades abordan los componentes integrales del derecho a la salud (Observación General 14, artículo 12, párrafo 3).

A su vez, para la Unicef,

las nuevas madres y los recién nacidos serán recibidos por una cruda realidad, y deberán enfrentarse a medidas de contención a nivel mundial, como los aislamientos y los toques de queda; el desbordamiento de los centros de salud debido a la respuesta a la enfermedad; la escasez de equipos y suministros; y una falta de parteras cualificadas, ya que los trabajadores sanitarios, incluidas las matronas, están siendo reubicados para tratar a los pacientes con covid-19. ${ }^{16}$

Relacionado con lo anterior, no debemos olvidar los factores propios de la planificación familiar, e incluir en este análisis a aquellas mujeres o parejas que no desean ser madres o padres. En este aspecto, es importante que exista una preocupación actual y efectiva a nivel país, a partir de los lineamientos internacionales en el asesoramiento y apoyo permanente a hombres, mujeres y parejas en el acceso y acompañamiento en tratamientos de fertilidad y control de natalidad, como los métodos anticonceptivos; en el proceso de embazado, parto y posparto y para aquellas mujeres que se encuentren dentro de las causales de despenalización del aborto y desean someterse a dicha intervención.

Es de imperiosa necesidad asegurar el acceso a servicios de salud o atención médica que garanticen una maternidad segura, libre de riesgos en los períodos de gestación, parto y lactancia, que brinde a los padres la máxima posibilidad de tener hijos sanos a causa de la pandemia y sus graves consecuencias. En período de cuarentenas y contagios, se debe evitar que los padres y el recién nacido se contagien al interior de los centros hospitalarios o del propio hogar.

Para la Naciones Unidas, «el covid-19 tendrá un impacto catastrófico para millones de mujeres. La falta de anticonceptivos provocará embarazos no queridos. Además, habrá un aumento de la violencia de género, los matrimonios infantiles y la mutilación femenina. Los países deben protegerlas». ${ }^{17}$

16. «Las mujeres embarazadas y los bebés nacidos durante la pandemia de la covid-19 se enfrentarán a unos sistemas de salud sobrecargados y a interrupciones en los servicios», Unicef, 7 de mayo de 2020, disponible en https://uni.cf/34rwbR1.

17. «Millones de mujeres sufrirán embarazos no deseados durante la pandemia de coronavirus», Noticias ONU, 28 de abril de 2020, disponible en https://bit.ly/3pazHyP. 
Es válido cuestionarse si en los tiempos que corren se pueden ejercer estas libertades de manera segura, cuando en la práctica las condiciones externas en los sistemas de salud y atenciones médicas están siendo visiblemente afectadas, por lo que deben asegurar todas las condiciones a las mujeres que decidan ejercer el derecho reconocido de interrupción legal en tres causales específicas, asumiendo como base la complejidad de la intervención desde lo biológico y sicológico, a lo que hay que agregar los confinamientos y cuarentenas, así como el miedo de contagio al verse en la necesidad de acceder a una intervención médica.

Según las recomendaciones internacionales provenientes del Royal College of Obstetrics and Gyneacology (RCOG) del Reino Unido, en el caso de la atención del aborto en el contexto de la pandemia del covid-19:

Para asegurar una atención del aborto segura y efectiva, podría requerirse atención a distancia o la realización de abortos con medicamentos de forma ambulatoria, especialmente en situaciones de aislamiento obligatorio o donde no se pueda acceder a una institución de salud [...]. La prioridad es la provisión de atención segura a las mujeres, incluyendo aquellas quienes son casos sospechosos o confirmados de covid-19. ${ }^{18}$

En temas de salud reproductiva, y a partir de una encuesta de la fundación MILES aplicada en línea a 514 personas, el Colegio Médico de Chile destaca las siguientes cifras:

$\mathrm{El} 72 \%$ de las mujeres que responden ha visto dificultado el acceso a anticonceptivos desde que llegó el Coronavirus a Chile. El 34\% refiere que no encontró stock de su anticonceptivo, 34\% denunció que el recinto de salud no estaba prestando el servicio y $30 \%$ vio aumentado el precio de los anticonceptivos. También, la encuesta reporta que 1 de 4 mujeres tuvo dificultades para acceder al PAP, y de las mujeres que intentaron consultar por infecciones de transmisión sexual, $80 \%$ no pudo acceder a la atención. ${ }^{19}$

Para reforzar lo dicho, la directora ejecutiva de Unicef, Henrietta Fore, recalca la nueva realidad de muchas madres y parejas que en un contexto de covid-19 deben enfrentar nuevos desafíos y riesgos asociados a la maternidad y crianza. Destaca que

millones de madres de todo el mundo iniciaron la aventura de la maternidad en el mundo que conocíamos. Ahora han de prepararse para traer una vida a un mundo totalmente distinto: un mundo en el que las futuras madres temen ir a los centros de salud por miedo a infectarse y en el que no pueden recibir cuidados de emergencia

18. Royal College of Obstetrics and Gyneacology, «Infección por covid-19 y atención al aborto», Red de Acceso al Aborto Seguro, 24 de abril de 2020, p. 2, disponible en https://bit.ly/34tyOlc.

19. Colegio Médico de Chile, «Género...», p. 10. 
debido a la sobrecarga de los servicios de la salud y los aislamientos [...] Es difícil hacerse una idea de hasta qué punto la pandemia del coronavirus ha transformado la maternidad. ${ }^{20}$

\section{Protección de las personas con $\mathrm{VIH} / \mathrm{sida}$}

Los derechos sexuales y el reconocimiento de la salud sexual y reproductiva provienen de aquella decisión que como personas ejercemos, según el grado de desarrollo progresivo tanto físico como mental que se evidencia en las diversas etapas la vida, y que se traducen en elegir libre y responsablemente si se desea o no ejercer una vida sexual activa, exigiendo del resto de la sociedad un pleno respeto por la integridad física del cuerpo y sus expresiones sexuales.

Se plantea la necesidad de proteger la salud sexual, sin postergar o descuidar su atención en períodos de covid-19. En estos términos, la Organización Mundial de la Salud ha establecido lineamientos referenciales en la materia, recomendando para el restablecimiento de las actividades y servicios de manera segura

velar por que se adopten estrategias de extensión específicas si la cobertura y el recurso a la atención sanitaria disminuyen. Prever las visitas prenatales pendientes, incluida la administración de vacunas con anatoxina tetánica y las pruebas del VIH y la sífilis. Establecer mecanismos para garantizar la concertación temprana y continua de las consultas o tratamientos que hayan quedado pendientes. Planificar la manera de poner al día los datos registrados en los hogares que estén incompletos. ${ }^{21}$

Resulta preocupante, teniendo en cuenta las recomendaciones de la OEA, que personas con VIH/sida puedan acceder a prestaciones y servicios de salud, evitando un actuar discriminatorio producto de la enfermedad que padecen:

El estigma que rodea al VIH (que en algunos contextos además se suma a la criminalización) hace que muchos no accedan a los testeos y, por consiguiente, a los tratamientos, y con ellos a poder convivir con el virus como una enfermedad crónica. Recordemos que las personas con VIH que no acceden a tratamientos y con ello a la indetectabilidad del virus en la sangre y control de sus $\mathrm{Cd}_{4}$, están más expuestos a la muerte por coronavirus. ${ }^{22}$

En refuerzo de lo anterior, la Comisión Interamericana de Derechos Humanos, en su Resolución 1-2020, sobre pandemia y derechos humanos en las Américas, hace un llamado a los Estados a enfocar sus esfuerzo en supervisar que los protocolos médi-

\footnotetext{
20. Unicef, «Las mujeres...».

21. «Mantenimiento de los servicios de salud esenciales: La guía operativa para el contexto de covid-19", Organización Mundial de la Salud, 1 de junio de 2020, p. 24, disponible en https://bit.ly/3rceVJ5. 22. Organización de los Estados Americanos, «Guía...», 49-50.
} 
cos, las decisiones sobre recursos médicos y tratamientos en relación al covid-19 sean aplicados sin discriminación en razón de la edad, y con especial atención aquellas personas mayores con discapacidad o condiciones crónicas y enfermedades, pacientes con VIH o sida, entre otras. Naciones Unidas advierte en el mismo tenor que la reasignación de los recursos sanitarios por parte de los Estados, en los mecanismos para prevenir y reaccionar ante el covid-19, ha creado o agravado la escasez de antirretrovirales para las personas que viven con el VIH. ${ }^{23}$

En Chile se han tomado medidas que garantizan y refuerzan las medidas aplicables para personas que viven con VIH/sida, recalcando el hecho de que deben tomar todas las precauciones para proteger y consultar de manera rápida y anticipada, en caso de presentar síntomas de covid-19, por lo que se vuelve imperioso potenciar los pilares necesarios en el acceso de tratamiento y atención médica.

El Ministerio de Salud de Chile, dada la situación actual a causa del covid-19 en el país, determinó que los centros de atención de VIH asegurarán la entrega de los tratamientos de cada persona en control y de preservativos, procurando la realización de un control médico periódico para aquellas personas con VIH que se mantienen estables y en tratamiento exitoso. ${ }^{24}$

Del análisis en este capítulo, se demuestra que a pesar de ser válidos y comprometidos los esfuerzos desarrollados por nuestro país en la garantía de los derechos esenciales, el camino para prevenir y combatir las consecuencias del covid-19 en los diversos aspectos de la vida de una persona es una tarea diaria que no debe darse por finalizada, asumiendo que los efectos de la pandemia permanecerán con nosotros en el mediano plazo. Por tanto, se reitera la necesidad de alinear y redoblar esos esfuerzos, en concordancia con las recomendaciones de los organismos internacionales, en la promoción y resguardo de la vida, la salud y la dignidad de todas las personas que habitan un planeta atacado por el covid-19.

\section{Reflexiones finales}

Luego de describir diversas situaciones de afectación y mayor exposición en el ejercicio de los derechos sexuales y reproductivos en diferentes grupos de la población a causa de la pandemia del covid-19, los Estados, como medida preventiva y reactiva ante una situación de emergencia sanitaria, con miras a la protección de la salud humana, deben observar y recoger las recomendaciones que los organismos internacionales hacen en la materia, con la finalidad de reforzar las garantías de aquellos derechos, materializándolo en políticas públicas concretas y eficientes; no basta con

23. Oficina del Alto Comisionado de Naciones Unidas para los Derechos Humanos, «El sufrimiento...».

24. «Información sobre covid-19 para personas que tienen VIH», Ministerio de Salud de Chile, 2020, disponible en https://bit.ly/3nwLKie. 
conocer los lineamientos internacionales si no vienen acompañados de medidas reales. Es por ese motivo que las autoridades no deben desproteger a aquellos grupos en situación de vulnerabilidad producto de la pandemia, cuyas realidades pueden verse desmedradas como consecuencia de los confinamientos y cuarentenas, lo que provoca situaciones hostiles, que deban ser intervenidas con urgencia, atendiendo la realidad de cada grupo social desde su especificidad.

A veinticinco años de la IV Conferencia de la Mujer de Beijín el año 1995, el 8 de marzo de 2020, el Administrador del Programa de las Naciones Unidas para el Desarrollo, Achim Steiner, informó:

En consonancia con el visionario programa de la Declaración y Plataforma de Acción de Beijing, el PNUD seguirá trabajando estrechamente con la familia de las Naciones Unidas, en particular con ONU Mujeres, para ayudar a promover una nueva generación de leyes, políticas y programas innovadores orientados a cambiar las creencias y prácticas discriminatorias, a fin de lograr la igualdad de género. ${ }^{25}$

Se valoran los lineamientos y medidas tomados por la comunidad internacional no sólo en la protección de los derechos sexuales y reproductivos de la mujer, sino que de toda persona, sea niña, niño, adolescente, parejas y grupos vulnerables o en circunstancias especiales, como son las personas con capacidades diferentes, provenientes de pueblos originarios o etnias indígenas, adultos mayores o personas que padecen enfermedades crónicas, etcétera.

Sin embargo - como lo hemos explicado durante el desarrollo de este trabajo-, en la actualidad la labor de fomentar, promover y proteger los derechos sexuales y reproductivos dice relación con una tarea mancomunada de los diversos actores del mundo social, político, cultural y sobre todo jurídico. Debemos lograr que los principios y normas esenciales emanados de la dignidad de las personas se concreten en políticas y medidas nacionales dentro de los Estados, acompañados de un avance progresivo en la mirada país de estos temas.

Se deben acoger y reforzar en los Estados las recomendaciones que los organismos internacionales hacen de manera firme, demostrando una preocupación a nivel mundial por los grupos más expuestos a las consecuencias del covid-19, con un llamado sólido y categórico a prestar atención en las medidas implementadas, en conjunto con aquellas destinadas a prevenir y enfrentar la emergencia sanitaria. Se debe evitar que las consecuencias graves del covid-19 provoquen de manera adicional situaciones más abrumadoras, que potencien los efectos negativos de la pandemia.

25. Achim Steiner, «Día Internacional de la Mujer 2020», Programa de las Naciones Unidas para el Desarrollo, 5 de marzo de 2020, disponible en https://bit.ly/37vin3B. 


\section{Referencias}

CorrêA, Sonia y Maria Betânia Ávila (2003). «Direitos sexuais reprodutivos: pauta global e percursos brasileiros». En Elza Berquó (editora), Sexo \& vida: Panorama da saúde reprodutiva no Brasil (pp. 17-78). Campinas: UNICAMP.

IIDH, Instituto Interamericano de Derechos Humanos (2008). Los derechos reproductivos son derechos humanos. San José: IIDH. Disponible en https://bit.ly/2JrRg6u.

Petchesky, Rosalind Pollack (1999). «Direitos sexuais: Um novo conceito na prática política internacional». En Richard Parker y Regina Maria Barbosa (editores), Sexualidades pelo avesso: Direitos, identidades e poder (pp. 15-38). Río de Janeiro: Editora 34 .

Saeteros, Rosa del Carmen, Giselda Sanabria y Julia Pérez (2014). «Imaginario sobre la homosexualidad en estudiantes de politécnicos ecuatorianos». Revista $\mathrm{Cu}$ bana Salud Pública, 40 (4): 299-313. Disponible en http://ref.scielo.org/5vxwbc.

\section{Sobre la autora}

Alejandra Isabel Pérez Ceballos es abogada y licenciada en Ciencias Jurídicas de la Universidad Bernardo O’Higgins, Chile. Magíster en Derecho Público en la Facultad de Derecho de la Universidad de Chile. Profesora de Derecho Constitucional en la Universidad Autónoma de Chile. Su correo electrónico es alejandra.perez. abogado@gmail.com. (D) http://orcid.org/0ooo-0001-9350-0888. 
El Anuario de Derechos Humanos es una publicación semestral de referencia y consulta en materia de derechos humanos y campos afines. Busca ser un espacio de discusión de los temas centrales en el ámbito nacional e internacional sobre derechos humanos. Es publicado desde 2005 por el Centro de Derechos Humanos de la Facultad de Derecho de la Universidad de Chile.

\author{
EDITORA \\ Claudia Iriarte Rivas \\ ciriarter@derecho.uchile.cl \\ SITIO WEB \\ anuariocdh.uchile.cl \\ CORREO ELECTRÓNICO \\ anuario-cdh@derecho.uchile.cl \\ LICENCIA DE ESTE ARTÍCULO \\ Creative Commons Atribución Compartir Igual 4.o Internacional
}

\author{
\% \\ La edición de textos, el diseño editorial \\ y la conversión a formatos electrónicos de este artículo \\ estuvieron a cargo de Tipográfica \\ (www.tipografica.io)
}

